\title{
Remarks on Light Scattering from Polymers in Mixed Solvents: Effects of Polymer Molecular Weight, Molecular Weight Distribution, and Solvent Composition
}

\author{
Edward F. CAsAssa \\ Department of Chemistry and Center for Special Studies, \\ Carnegie-Mellon University, Pittsburgh, Pennsylvania 15213, U.S.A.
}

(Received November 13, 1971)

\begin{abstract}
It is known that light-scattering data for a polymer in a mixed solvent together with the refractive-index increment of the polymer evaluated at osmotic equilibrium (i.e., at fixed chemical potentials of solvent components) permit unambiguous determination of the molecular weight of the polymer-irrespective of preferential interactions between polymer and solvent. However, the analysis presumes either of two conditions; the polymer is homogeneous in molecular weight or the "osmotic" refractive increment does not depend on molecular weight. When both conditions are violated, the apparent molecular weight obtained may differ from the true weight average. This problem is examined with the aid of parameters derived from light-scattering data of Dondos and Benoît. The error in typical cases is shown to be less than uncertainty in scattering measurements if polymer heterogeneity is not extreme (say $M_{w} / M_{n} \leq 2$ ); but the behavior of each scattering system must be assessed individually. The data also afford material for some observations on the dependence of preferential interactions on composition of a binary solvent.
\end{abstract}

KEY WORDS Light Scattering / Refractive-Index Increment / Mixed Solvent / Preferential Interaction / Apparent Molecular Weight / Molecular Distribution /

In a number of studies concerning macromolecular solutes in mixed solvents, it has been shown that knowledge of the refractive-index increment for the solute evaluated at osmotic equilibrium between solution and solvent affords a notable simplification of the analysis of light-scattering data and permits molecular weights to be obtained unambiguously, without regard to preferential thermodynamic interactions between polymer and a solvent component (or components). ${ }^{1-4}$ If, however, the polymer is heterogeneous in molecular weight and if the amount of solvent preferentially "bound" per unit mass of polymer depends on molecular weight, complications arise. In the following discussion, which elaborates on brief comments given elsewhere, ${ }^{5}$ we consider this problem and offer a quantitative assessment of the effect on molecular weight determinations in some typical cases. The data at hand also prompt some remarks on the dependence of preferential interaction on composition of a binary solvent.

If certain physical approximations are valid,
Rayleigh scattering of radiation from a solution of a single macromolecular species (component 2 ) in a pure solvent (component 1) is expressed by the familiar relation ${ }^{6}$

$$
K^{\prime}\left(\partial n / \partial c_{2}\right)_{P}{ }^{2} c_{2} / R_{0}=M_{2}^{-1}+2 A_{2} c_{2}+0\left(c_{2}{ }^{2}\right)
$$

where $K^{\prime}$ denotes a combination of optical and numerical constants; $c_{2}$ is the concentration (weight/volume) of solute and $M$ its molecular weight; $R_{0}$ is the reduced scattered intensity (scattering from solution less that from solvent) extrapolated to zero scattering angle; $n$ is the refractive index of the solution; and $A_{2}$ is the second virial coefficient in the expansion of the equation of state in powers of solute concentration.

If component 2 is dissolved in a mixture of two solvents (components 1 and 3 ) the theory for scattering from multicomponent systems ${ }^{1-4,7}$ leads to a relation of the same form as eq 1 ;

$$
K^{\prime}\left(\partial n / \partial c_{2}\right)_{P, T, \mu_{3}}^{2} c_{2} / R_{0}=M^{-1}+2 A_{2} c_{2}+O\left(c_{2}{ }^{2}\right)
$$

with the refractive-index increment $\left(\partial n / \partial c_{2}\right)_{P, T, \mu_{3}}$ 


\section{E. F. CASASSA}

evaluated at fixed pressure, temperature, and chemical potential $\mu_{3}$ of component 3. Writing the refractive index as a function of $c_{2}$ and composition of mixed solvent (e.g., volume fraction $\phi_{3}$ of component 3 in the binary solvent mixture) we obtain

$$
\left(\frac{\partial n}{\partial c_{2}}\right)_{P, T, \mu_{3}}=\left(\frac{\partial n}{\partial c_{2}}\right)_{P, T, \phi_{3}}+\lambda\left(\frac{\partial n}{\partial \phi_{3}}\right)_{P, T, c_{2}}
$$

where the quantity

$$
\lambda=\left(\frac{\partial \phi_{3}}{\partial c_{2}}\right)_{P, T, \mu_{3}}
$$

represents the thermodynamic interaction between solute and solvent $3 .^{7,8}$ A positive $\lambda$ can be thought of as signifying preferential solvation of solute by solvent 3 or, equivalently, rejection of solvent 1 by the solute. For ordinary solutions ${ }^{4}$ it happens that, except for inconsequential error,

$$
\left(\frac{\partial n}{\partial c_{2}}\right)_{P, \mu_{3}}=\left(\frac{\partial n}{\partial c_{2}}\right)_{\mu_{1}, \mu_{3}} \equiv\left(\frac{\partial n}{\partial c_{2}}\right)_{\mu}
$$

(To simplify notation slightly we suppress the subscript $T$ and use the single subscripts $\mu$ to indicate constancy of the potentials of both solvent components and $\phi$ to indicate a fixed solvent composition.)

Equation 5 shows that the refractive increment needed to permit direct determination of the molecular weight $M$ from scattering data according to eq 2 can be obtained by refraction measurements on solutions equilibrated by dialysis against the mixed solvent of composition $\phi_{3}$. This possibility is of evident importance if the polymer cannot be dissolved in a single solvent. From another point of view, the coefficient $\lambda$ is itself of interest and can be determined in various ways; by comparing $\left(\partial n / \partial c_{2}\right)_{\mu}$ and $\left(\partial n / \partial c_{2}\right)_{\phi}$ if $\left(\partial n / \partial \dot{\phi}_{3}\right)_{c_{2}}$ is known; from a known $M$ and lightscattering data together with $\left(\partial n / \partial c_{2}\right)_{\phi}$ and $\left(\partial n / \partial \phi_{3}\right)_{c_{2}}$; or from direct chemical analysis of solution and pure solvent after equilibration by dialysis.

The generalization to a solvent of many components is straightforward and will not be discussed here.

\section{SCATTERING FROM A HETEROGENEOUS POLYMER IN A MIXED SOLVENT}

The above relations hold for a homogeneous solute, but since typical polymer samples exhibit significant dispersion in molecular weight, the effect of this has to be considered. Now we let the solute comprise a number of components $J$ differing in molecular weight $M_{J}$, the total concentration being

$$
c=\sum_{J} c_{J}=c \sum_{J} w_{J}
$$

where $w_{J}$ is the weight fraction of polymer $J$ in the solute mixture. If there is but one solvent, and if the refractive increment $\partial n / \partial c_{J}$ is the same for every solute, the form of eq 1 can still be written, as is well known, in terms of the total solute concentration $c$ and the weight-average molecular weight $M_{w}{ }^{6}$

For the heterogeneous solute in a binary solvent mixture, contributions to $R_{0}$ due to the presence of each solute species are additive as $c \rightarrow 0$ : i.e.,

$$
R_{0}=K^{\prime} \sum_{J} M_{J}\left(\partial n / \partial c_{J}\right)_{\mu}{ }^{2} c_{J}+O\left(c^{2}\right)
$$

Then, if the reciprocal scattering function is formulated in terms of the experimental refractive increment

$$
\left(\frac{\partial n}{\partial c}\right)_{\mu}=\sum_{J}\left(\frac{\partial n}{\partial c_{J}}\right)_{\mu} w_{J}
$$

for the heterogeneous solute, eq 1 is replaced by

$$
\lim _{c \rightarrow 0}\left[\frac{K^{\prime}(\partial n / \partial c)_{\mu}{ }^{2} c}{R_{0}}\right]=\frac{(\partial n / \partial c)_{\mu}{ }^{2}}{\sum_{J}\left(\partial n / \partial c_{J}\right)_{\mu}{ }^{2} M_{J} w_{J}} \equiv \frac{1}{M_{w}{ }^{+}}
$$

If $\left(\partial n / \partial c_{J}\right)_{\mu}$ is independent of molecular weight $M_{J}$, the refractive increments on the right hand side of eq 8 cancel, and a plot of the scattering function versus concentration $c$ extrapolates to the reciprocal of $M_{w}$. Otherwise, this procedure gives an apparent average molecular weight $M_{w}{ }^{+}$ defined by eq 9 . Obviously, dependence of

$$
\left(\frac{\partial n}{\partial c_{J}}\right)_{\mu}=\left(\frac{\partial n}{\partial c_{J}}\right)_{\phi}+\lambda_{J}\left(\frac{\partial n}{\partial \phi_{3}}\right)_{c_{J}}
$$

on molecular weight of solute can arise from variation with molecular weight in $\left(\partial n / \partial c_{J}\right)_{\phi}$ and/or in the interaction coefficient $\lambda_{J}$. Presuming that any variation in $\left(\partial n / \partial c_{J}\right)_{\dot{\phi}}$ is too small to be important at reasonably high molecular weight, we proceed to consideration of $\lambda$. 


\section{DEPENDENCE OF PREFERENTIAL INTERACTIONS ON MOLECULAR WEIGHT OF POLYMER}

Various assumptions about the behavior of $\lambda$ with molecular weight of a long-chain homopolymer might be contemplated. Three possibilities are the following:

(I) The interaction expressed by $\lambda$ can be associated with "sites" on the polymer chain in the sense that a certain average number of molecules of solvent 3 is preferentially bound by each monomer residue in the chain. Hence $\lambda$ does not depend on the molecular weight of the polymer. ${ }^{4}$

(II) Assumption I is correct as a first approximation, but the picture should be modified by recognizing that intramolecular contacts between chain segments may affect polymer-solvent contacts with something like one-to-one equivalence. Assuming further that the dissolved polymer chain can be represented by a Gaussian distribution of chain segments with its mean-square radius of gyration proportional to the molecular weight and that the probability of occurrence of polymer-polymer contacts in any part of the chain domain is proportional to the square of the local segment density, it can be shown ${ }^{9,10}$ that the average number of intersegmental contacts per chain segment varies inversely with $M^{1 / 2}$. Hence $\lambda$ depends linearly on $M^{-1 / 2}$ :

$$
\lambda / \lambda_{\infty}=1+A M^{-1 / 2}
$$

where $\lambda_{\infty}$ is the limiting value of $\lambda$ at high molecular weight. However, since the dissolved polymer chain is in effect a rather dilute solution of monomer units (e.g., a mole fraction on the order of 0.01 within the chain domain) intramolecular contacts are rare compared with polymer-solvent contacts; and the second term on the rhs of eq 11 is of little consequence except at low molecular weight. ${ }^{9}$

(III) The preferential solvent "binding" is represented by eq 11 , but the second term may be larger than would be expected on the basis of any simple equivalence between intramolecular contacts and polymer-solvent contacts.

From a recent experimental study by Dondos and Benoît, ${ }^{10}$ it appears that statement III is reasonable. Using a series of narrow-distribution polystyrenes prepared anionically, they compared the light-scattering molecular weight measured in a single solvent with the apparent molecular weight $M^{*}$ obtained by applying eq 1 to data from the same polymer in a binary solvent:

$$
\frac{1}{M^{*}} \equiv \lim _{c_{2}=0}\left[\frac{K^{\prime}\left(\partial n / \partial c_{2}\right)_{\phi}{ }^{2}}{R_{0}}\right]=\frac{1}{M}\left[1+\lambda \frac{\left(\partial n / \partial \phi_{3}\right)_{c_{2}}}{\left(\partial n / \partial c_{2}\right)_{\phi}}\right]^{-2}
$$

Calculating $\lambda$ from eq 12 (and known values of $\left(\partial n / \partial \dot{\phi}_{3}\right)_{c}$ and $\left.\left(\partial n / \partial c_{2}\right)_{\phi}\right)$ they plotted $\lambda M^{1 / 2} v s . M^{1 / 2}$



Figure 1. Preferential interactions in three-component solutions: polystyrene-solvent(1)-solvent (3). Plots of $\left(M^{*} / M\right)^{1 / 2}$ vs. $M$ for solvent mixtures listed in Table I: systems 1, 2, 3, and 4, [benzene (1) + methanol(3)]; system 9, [benzene(1)+heptane (3)]; system 10, [dioxane(1)+methanol(3)].

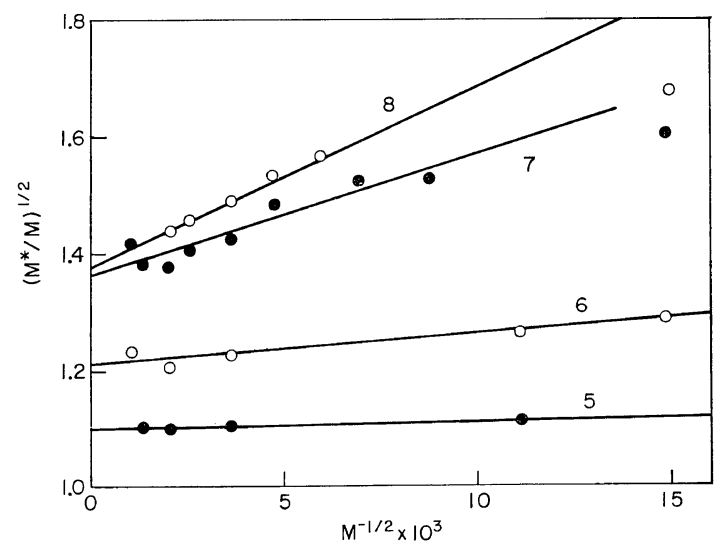

Figure 2. Plots as in Figure 1: systems 5, 6, 7, and 8 , [carbon tetrachloride (1)+methanol(3)]. 


\section{E. F. CASASSA}

Table I. Light scattering from polystyrene in mixed solvents

(Parameters derived from data of Dondos and Benoît ${ }^{10}$ )

\begin{tabular}{|c|c|c|c|c|c|c|}
\hline System & Solvent & $\phi_{3}$ & $-\mathrm{d} n / \mathrm{d} \phi_{3}^{\mathrm{a}, \mathrm{b}}$ & $(\partial n / \partial c)_{\dot{\phi}}^{\mathrm{b}}$ & $-\lambda_{\infty}$ & $A$ \\
\hline 1 & Benzene $(1)+$ methanol $(3), 25^{\circ} \mathrm{C}$ & 0.100 & 0.171 & 0.123 & $0.13 \pm 0.09$ & $22 \pm 15$ \\
\hline 2 & & 0.190 & " & 0.140 & $0.34 \pm 0.02$ & $30 \pm 7$ \\
\hline 3 & & 0.220 & " & 0.145 & $0.351 \pm 0.006$ & $53 \pm 4$ \\
\hline 4 & & 0.255 & " & 0.150 & $0.434 \pm 0.007$ & $50 \pm 1$ \\
\hline 5 & Carbon tetrachloride $(1)+$ methanol $(3), 25^{\circ} \mathrm{C}$ & 0.050 & 0.131 & 0.150 & $0.111 \pm 0.002$ & $13 \pm 3$ \\
\hline 6 & & 0.100 & $" 1$ & 0.154 & $0.242 \pm 0.009$ & $24 \pm 6$ \\
\hline 7 & & 0.185 & " & 0.162 & $0.44 \pm 0.01$ & $58 \pm 3$ \\
\hline 8 & & 0.210 & " & 0.164 & $0.465 \pm 0.004$ & $83 \pm 2$ \\
\hline 9 & Benzene $(1)+$ heptane $(3), 25^{\circ} \mathrm{C}$ & 0.540 & 0.112 & 0.160 & $0.176 \pm 0.003$ & $47 \pm 2$ \\
\hline 10 & Dioxane $(1)+$ methanol(3), $35^{\circ} \mathrm{C}$ & 0.050 & 0.0873 & 0.202 & $0.03 \pm 0.01$ & $250 \pm 100$ \\
\hline
\end{tabular}

a Values used by Dondos and Benoît (obtained by approximating $n$ as a linear function of composition of solvent by volume).

b Refractive increments for $5461 \AA$.

for ten ternary systems to obtain $\lambda_{\infty}$ and the molecular weight dependence of $\lambda$, respectively, from slopes and intercepts of the best fitting straight lines.

In Figures 1 and 2 we have plotted the data of Dondos and Benoît as $\left(M^{*} / M\right)^{1 / 2}$ vs. $M^{-1 / 2}$, a form that perhaps exhibits more clearly the molecular weight dependence of $\lambda$ and the experimental uncertainty in the data. From the least-squares straight lines we obtained the values of $\lambda_{\infty}$ and $A$ recorded in Table I. The calculation of $\lambda$ requires knowledge of $\left(\partial n / \partial c_{2}\right)_{\phi}$ and of $(\partial n / \partial \phi)_{c_{2}}$ at $c_{2}=0$. The first quantity is given by Dondos and Benoit, and the values they used for the second are implied by values of $M^{*}$ and $\lambda$ that they list. The probable errors ${ }^{11}$ in the parameters are included to give some idea of the adequacy of the linear fitting of the data points.

It is clear that eq 11 provides an adequate representation of the experimental data within the experimental uncertainty, although a slight upward convexity in some of the plots__- particularly those for systems 3 and 4 suggests a possible dependence of $\lambda$ on $M^{-a}$ with $a<1 / 2$. The points at lowest molecular weight (4500) were omitted in computing the least-squares parameters; thus the values of $A$ reflect, predominantly, molecular weight dependence of $\lambda$ for molecular weights on the order of $10^{4}$ and above. Although Dondos and Benoît do not tabulate $\lambda_{\infty}$, or a parameter characterizing the molecular weight dependence of $\lambda$, it is obvious that the results obtained here are similar to theirs. Predictably, the different data analysis carried through here cannot alter the character of the results though it does produce some quantitative differences. Our $\lambda$ 's, unlike those of Dondos and Benoît, are negative: this reflects nothing more than our designation of the poor solvent, the minor component in all but one of the binary mixtures, as component 3 .

\section{DEPENDENCE ON SOLVENT COMPOSITION}

In Figure 3, the dependence of $\lambda_{\infty}$ on solvent composition is exhibited in a plot that would be appropriate if solvent binding could be described in terms of sites on the polymer molecules that compete for the solvent components in such fashion that

$$
\frac{\dot{\phi}_{3}{ }^{\prime \prime}}{{\phi_{1}}^{\prime \prime}}=K \frac{\phi_{3}}{\phi_{1}}
$$

where $\phi_{1}{ }^{\prime \prime}$ and ${\phi_{3}}^{\prime \prime}$ are volume fractions of components 1 and 3 in the "bound" solvent material. Accordingly, preferential interaction with component 3 is positive or negative respectively as $K$ is greater or less than unity. It can be shown (see Appendix A) that the plots of $\phi_{1} \phi_{3} / \lambda$ in Figure 3 will be linear if eq 13 is obeyed. The data do in fact fall roughly on straight lines; but it would be unwarranted on this tenuous basis to conclude that the occurrence of site binding has been demonstrated. We can look upon $\phi_{1} \phi_{3} / \lambda$ simply as a possibly useful function 




Figure 3. Dependence of $\lambda$ on solvent composition for polystyrene in mixed solvents at $25^{\circ} \mathrm{C}$ : plot $\mathrm{A}$, [benzene(1)+methanol(3)]; plot $\mathbf{B}$, [carbon tetrachloride(1) + methanol(3)] from recalculation of data of Dondos and Benoît ${ }^{10}$; plot $\mathrm{C}$, ordinate at right, [benzene(1)+cyclohexane(3)] from data of $\operatorname{Read}^{9}$ (open squares) and data of Strazielle and Benoît ${ }^{11}$ (open circles).

that is well behaved at the limits $\phi_{3}=0$, and $\phi_{3}=1$ where we expect $\lambda$ to be zero and $\mathrm{d} \lambda / \mathrm{d} \phi_{3}$ to be finite (see Appendix B). Obviously, this form and the model suggesting it cannot be applicable when, as has been reported ${ }^{12-14}$ in a few instances, $\lambda$ changes sign at some $\phi_{3}$.

For comparison we have included in Figure 3 some data from two studies of preferential interactions in polystyrene-benzene(1)-cyclohexane(3) mixtures covering a wide range of solvent composition. According to data of $\operatorname{Read}^{9} \phi_{1} \phi_{3} / \lambda$ varies weakly with $\phi_{3}$. The results of Strazielle and Benoit ${ }^{15}$ indicate a similar, possibly more pronounced, dependence on $\phi_{3} .{ }^{*}$ Read obtained good agreement between his experimental $\lambda$ and a more complicated dependence on composition derived by extending a Flory-Huggins latticetheory formulation to include ternary interaction parameters. Other experimental studies of $\lambda$ and its variation with solvent composition are summarized in a review by Cowie. ${ }^{13}$

* Volume fraction compositions in Table I and Figure 3 are based on volumes of the unmixed components. Use of the theoretically preferable magnitudes computed from partial volumes (see Appendix A) would not materially affect our analysis.

\section{EFFECT OF MOLECULAR WEIGHT DISTRIBUTION ON $M_{w}{ }^{+}$}

Using numerical parameters derived from the data of Dondos and Benoît, ${ }^{10}$ we turn now to a calculation of $M_{w}{ }^{+}$for a heterogeneous polymer in a binary solvent. Since we have to assume some specific form for the molecular weight distribution, completely general conclusions cannot be inferred from the results, but they will provide some guidance as to whether in typical circumstances $M_{w}^{+}$can be identified with $M_{w}$ without important error.

Because it is easy to manipulate mathematically, we employ the Schulz ${ }^{16}$ distribution, described for a continuous distribution of molecular weight $M$, by

$$
f(M)=\left(\frac{Z+1}{M_{w}}\right)^{Z+1} \frac{M^{Z}}{\Gamma(Z+1)} \exp \left\{-\frac{(Z+1) M}{M_{w}}\right\}
$$

where $F(M) \mathrm{d} M$ is the weight fraction of polymer in the differential increment $\mathrm{d} M$, and $\Gamma$ denotes the gamma function. With $Z=1$, eq 14 reduces to the "most probable" distribution produced by equilibrium condensation processes and certain vinyl polymerizations. Combining eq $3,5,8,11$, and 14 we find

$$
\begin{aligned}
\left(\frac{\partial n}{\partial c}\right)_{\mu} & =\left(\frac{\partial n}{\partial c}\right)_{\phi}+\lambda_{\infty}\left(\frac{\partial n}{\partial \phi_{3}}\right) \int_{0}^{\infty}\left(1+\frac{A}{M^{1 / 2}}\right) f(M) \mathrm{d} M \\
& =B\left[1+Y \frac{(Z+1)^{1 / 2} \Gamma(Z+1 / 2)}{\Gamma(Z+1)}\right]
\end{aligned}
$$

where

$$
B=\left(\frac{\partial n}{\partial c}\right)_{\phi}+\lambda_{\infty}\left(\frac{\partial n}{\partial \dot{\phi}_{3}}\right)_{c}
$$

and

$$
Y=\frac{A \lambda_{\infty}}{B M_{w}^{1 / 2}}\left(\frac{\partial n}{\partial \phi_{3}}\right)_{c}
$$

Similarly we obtain

$$
\begin{aligned}
\frac{1}{M_{w}} & \sum_{J}\left(\frac{\partial n}{\partial c_{J}}\right)_{\mu}^{2} M_{J} w_{J} \\
& =\frac{1}{M_{w}} \int_{0}^{\infty}\left[B+\lambda_{\infty}\left(\frac{\partial n}{\partial \phi_{3}}\right)_{c} \frac{A}{M^{1 / 2}}\right]^{2} M f(M) \mathrm{d} M \\
& =B^{2}\left[1+2 Y \frac{\Gamma(Z+3 / 2)}{(Z+1)^{1 / 2} \Gamma(Z+1)}+Y^{2}\right]
\end{aligned}
$$

Then, substitution of eq 15 and 18 into eq 9 


\section{E. F. CAsassa}

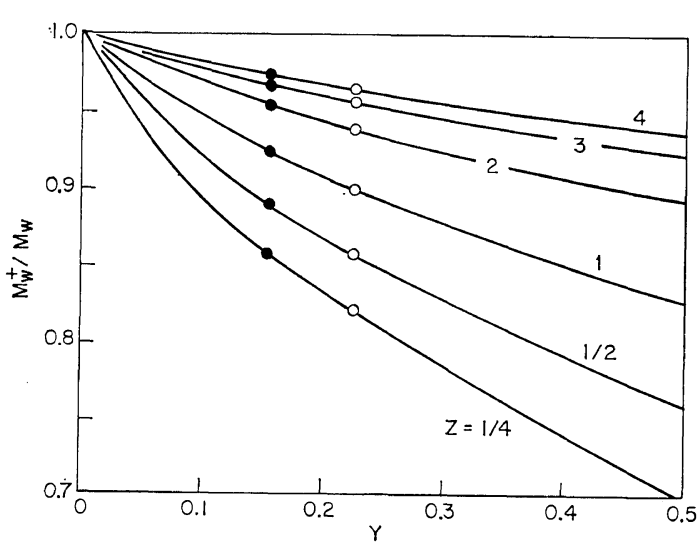

Figure 4. Effect of polymer heterogeneity and molecular weight dependence of $\lambda$ on apparent molecular weight $M_{w}^{+}$: plots of $M_{w}{ }^{+} / M_{w} v s$. $Y$ for values of the Schulz dispersity parameter $Z$ as indicated. The points mark values of $Y$ for $M_{w}=10^{4}$ and the parameters of system 7 (filled circles) and system 8 (open circles).

gives $M_{w}{ }^{+}$. For example, if $Z=1$, we have

$$
\frac{M_{w}{ }^{+}}{M_{w}}=\frac{1+1.878 Y+Y^{2}}{1+2.506 Y+1.571 Y^{2}}
$$

In Figure 4 we exhibit plots of $M_{w}{ }^{+} / M_{w}$ for the Schulz distribution for values of $Z$ between 0.25 and 4 (i.e., for weight-to-number-average molecular weight ratios from 5 to 1.25). The points shown on the graphs are for the parameters of Systems 7 and 8 in Table I and $M_{w}=10^{4}$. Analogous points for $M_{w}>10^{4}$ would lie nearer $Y=0$. In all the experimental systems discussed here, it happens that $(\partial n / \partial c)_{\phi}$ is positive and the solvent component of lower refractive index is selectively rejected by the polymer. Hence $Y$ is positive and $M_{w}{ }^{+}<M_{w}$, but physically there is no reason to exclude negative $Y$. Then we would have $M_{w}{ }^{+}>M_{w}$ at small $Y$ and an interesting consequence in the occurrence of a point at which $M_{w}{ }^{+}=M_{w}$ for some $Y<0$ for a given value of $Z$.

\section{DISCUSSION}

The solvent media listed in Table I each contain a good solvent for polystyrene and a precipitant, and were presumably chosen to exhibit marked preferential interactions. The ratios of the slopes to intercepts of the linear plots in
Figures 1 and 2 give $\left(A \lambda_{\infty} / B\right)\left(\partial n / \partial \phi_{3}\right)_{c}$ as a measure of the effect of the variations in $\lambda$ on $M^{*} / M$ for a homogeneous polymer and thus on $M_{w}{ }^{+}$ for a heterogeneous polymer. By this criterion System 8 shows the largest effect, and systems 3, 4 , and 7 fall next at about the same level. The data in Figure 2 suggest that the least-squares line for the points included in the calculation for system 8 may give an overestimate of the slope and that system 7 may be more reliably representative of the extreme effect in the systems studied by Dondos and Benoît. But even for the parameters of system 8 , a heterogeneous polymer having a Schulz distribution with $Z>1$ and $M_{w}>10^{4}$ will show a disparity between $M_{w}^{+}$and $M_{w}$ scarcely greater than the absolute uncertainty in molecular weights measured by light scattering (see Figure 4). We conclude that for high polymers with moderate distribution of molecular weight, and particularly for reasonably well-fractionated materials, $M_{w}$ can often be determined accurately by light scattering without independent knowledge of $\lambda$ if the refractive increment $(\partial n / \partial c)_{\mu}$ for the entire solute is determined. However, caution is necessary: $M_{w}{ }^{+}$is affected by $\lambda-$ by its magnitude and dependence on molecular weight and on the breadth and form of the molecular weight distribution__and by magnitudes of $(\partial n / \partial c)_{\phi}$ and $\left(\partial n / \partial \phi_{3}\right)_{c}$. It is not difficult to imagine situations in which $M_{w}{ }^{+}$might differ seriously from $M_{w}$. For example, a solvent mixture might be found such that $(\partial n / \partial c)_{\phi}$ vanishes to make $\left(\partial n / \partial c_{J}\right)_{\mu}{ }^{2}$ directly proportional to the molecular weight dependent $\lambda^{2}$; and of course an arbitrary molecular weight distribution could be chosen to maximize the effect of the variation of $\lambda$. Consequently the reassuring nature of the quantitative results obtained here provides no warrant for undiscriminatingly assuming that $M_{w}{ }^{+}$is indistinguishable from $M_{w}$.

Our assumption that the refractive-index increment $\left(\partial n / \partial c_{2}\right)_{\phi}$ is independent of molecular weight calls for some comment as this has been a matter of dispute. Since Dondos and Benoît found constant values for this quantity, we have made no provision for a variation with molecular weight. This is the familiar assumption in determination of weight-average molecular weights by light scattering in a single solvent; but it has been questioned recently, especially by measure- 
ments of Barrall, et al. ${ }^{17}$ who report refraction measurements on polystyrene-toluene and polystyrene-butanone systems covering a very wide molecular weight range. A fairly rapid change in $\mathrm{d} n / \mathrm{d} c_{2}$ at low molecular weights, say less than $10^{3}$ or even $10^{4}$, is hardly surprising since chain end-groups are of necessity different chemically from the rest of the molecule. The effect has been predicted realistically for polyalkanes ${ }^{18}$ on the assumption that molar refractions are constitutive and additive properties. However, the effect on $\mathrm{d} n / \mathrm{d} c_{2}$ that can be accounted for in this way is small for molecular weights above $10^{4}$. The magnitude of the molecular weight dependence observed at high molecular weights by Barrall, et al., is unexpectedly large and is not in agreement with data reported on other polymers. However, the limited amount of data available and the experimental uncertainty in all of it perhaps leave this matter in the realm of unresolved questions.

Whether or not a contribution from $\left(\partial n / \partial c_{2}\right)_{\phi}$ should be included in the molecular weight dependence of $\left(\partial n / \partial c_{2}\right)_{\mu}$, the observed dependence is so large in most of the systems studied by Dondos and Benoit that the effect certainly has to be ascribed primarily to the variation in preferential interaction. From a completely noncommittal point of view, however, Figures 1 and 2 can be taken simply as demonstrating empirically a linear relation between $\left(\partial n / \partial c_{2}\right)_{\mu}$ and $M^{-1 / 2}$. Then, the calculations for a heterogeneous polymer still give the effect of the variation of $\left(\partial n / \partial c_{2}\right)_{\mu}$ on $M_{w}{ }^{+}$: eq 15 and 18 remain applicable with $Y$ interpreted as $A^{\prime} / M_{w}{ }^{1 / 2}$ where $A^{\prime}$ is the constant in the relation

$$
\left(\frac{\partial n}{\partial c_{J}}\right)_{\mu}=\left(\frac{\partial n}{\partial c_{J}}\right)_{\mu, \infty}\left[1+\frac{A^{\prime}}{M_{J}^{1 / 2}}\right]
$$

Equations 10 and 11 constitute a special case of eq 20 with $A^{\prime}=A \lambda_{\infty}(\partial n / \partial \phi)_{c} /\left(\partial n / \partial c_{J}\right)_{\mu, \infty}$ if $\left(\partial n / \partial c_{J}\right)_{\phi}$ is independent of molecular weight.

To conclude, we reiterate that $M_{w}{ }^{+}$differs from the true weight average only if $\left(\partial n / \partial c_{2}\right)_{\mu}$ depends on molecular weight and at the same time the polymer is heterogeneous. At the limit of infinite dilution of polymer, eq 2 holds for every component $J$ in the solute. Then if $\left(\partial n / \partial c_{J}\right)_{\mu}$ is the same for all $J$, the weight-average molecular weight is obtained in the usual way according to eq 9. If $\left(\partial n / \partial c_{2}\right)_{\mu}$ depends on molecular weight of polymer, the correct weight for a homogeneous polymer is obtained from eq 2, but it is necessary to measure $\left(\partial n / \partial c_{2}\right)_{\mu}$ for the particular polymer under study.

Acknowledgements. This work was supported in part by the National Science Foundation under Research Grant No. GP-28538X. I am indebted also to Mrs. Aileen Liu for aid with calculations and to Dr. C. T. Greenwood, Flour Milling and Baking Research Association, Hertfordshire, for a discussion (by letter) that inspired this inquiry.

\section{APPENDIX A}

A linear dependence of $\phi_{1} \phi_{3} / \lambda$ on $\phi_{3}$ can be derived as follows. We imagine that 1 gram of polymer (component 2 ) in a solution at osmotic equilibrium with the binary solvent of volume fraction composition $\phi_{3}{ }^{\prime}$ binds $\xi_{1}$ grams of component 1 and $\xi_{3}$ grams of component 3. One $\mathrm{m} l$ of the ternary solution contains $c_{2}$ grams of polymer. The partial volume contribution due to the polymer is $c_{2} \bar{v}_{2}$ and that of bound material is $c_{2}\left(\xi_{1} \bar{v}_{1}+\xi_{3} \bar{v}_{3}\right)$ where $\bar{v}_{1}, \bar{v}_{2}, \bar{v}_{3}$ represent partial specific volumes of the components, assumed to be the same for bound and free solvent components. From the additivity of partial volumes it follows that the total mass of solvent 3 in one $\mathrm{m} l$ of the ternary system is

$$
c_{3}=c_{2} \xi_{3}+c_{3}^{\prime}\left[1-c_{2}\left(\bar{v}_{2}+\xi_{1} \bar{v}_{1}+\xi_{3} \bar{v}_{3}\right)\right]
$$

where $c_{3}{ }^{\prime}$ is the concentration of solvent $3(\mathrm{~g} / \mathrm{ml})$ in the binary solvent mixture equilibrated with the polymer solution (assuming that solution and solvent are incompressible). The volume fraction $\phi_{3}$ of component 3 in all the solvent material in the ternary system is

$$
\phi_{3}=c_{3} \bar{v}_{3} /\left(1-c_{2} \bar{v}_{2}\right)
$$

Combining eq A.1 and A.2, we obtain the difference in solvent composition between the two phases at equilibrium, and then passing to the limit as $c_{2}=0$ we find

$$
\begin{aligned}
\lim _{c_{2}=0}\left[\left(\phi_{3}-\phi_{3}{ }^{\prime}\right) / c_{2}\right] & =\left(\partial \phi_{3} / \partial c_{2}\right)_{\mu} \equiv \lambda \\
& =\xi_{3} \bar{v}_{3}-\phi_{3}\left(\xi_{1} \bar{v}_{1}+\xi_{3} \bar{v}_{3}\right)
\end{aligned}
$$

Now we assume that binding can be associated with $\nu$ sites per gram of polymer, each site capable of binding either $g_{1}$ grams of component 1 or $g_{3}$ 
grams of component 3 so that

$$
\frac{\xi_{1}}{g_{1}}+\frac{\xi_{3}}{g_{3}}=\nu
$$

Further, we assume that competitive binding is governed by an isotherm of the form

$$
\frac{\xi_{3} / g_{3}}{\xi_{1} / g_{1}}=k\left(\frac{\phi_{3}}{\phi_{1}}\right)
$$

Obviously, eq A.5 is symmetrical in the two solvent components, in that at the limits of mixtures dilute in either solvent 1 or 3 it represents the familiar Langmuir isotherm. In effect eq A.5 is a Langmuir expression for distribution of two components among equivalent independent sites, all of which must be occupied.

By combining eq $\mathrm{A} \cdot 4$ and $\mathrm{A} \cdot 5$ with eq $\mathrm{A} \cdot 3$ to eliminate $\xi_{1}$ and $\xi_{3}$, we obtain

$$
\lambda=\frac{\phi_{3}\left(1-\phi_{3}\right) I^{-1}}{1+(k-1) \phi_{3}}
$$

where

$$
I^{-1}=\nu k g_{3} \bar{v}_{3}-\nu g_{1} \bar{v}_{1}
$$

Rearrangement then gives $\phi_{1} \phi_{3} / \lambda$ as a linear function of $\phi_{3}$ with an intercept $I$ at $\phi_{3}=0$ and slope $(k-1) I$. Correspondence with eq 13 is obtained by letting $K=\bar{v}_{3} g_{3} k / \bar{v}_{1} g_{1}$.

\section{APPENDIX B}

The chemical potential $\mu_{J}$ of solute $J$ in a multicomponent solution can be expressed in the form

$$
\mu_{J}=\mu_{J}^{\circ}(P, T),+R T \ln m_{J}+\mu_{J}^{\mathrm{E}}
$$

where $m_{J}$ is the molality of component $J$ (moles per $\mathrm{kg}$ of principal solvent, component 1) and $\mu_{J}{ }^{\mathrm{E}}$ is the excess chemical potential. The laws of thermodynamics impose no particular form on the excess potential but experience with nonelectrolyte solutions shows that a very large class of mixtures are well behaved in the sense that $\mu_{J}^{\mathrm{E}}$ can be expanded in integral positive powers of concentration:

$$
\mu_{J}^{\mathrm{E}} / R T=\sum_{K=2,3} \beta_{J K} m_{K}+\sum_{K} \sum_{L} \gamma_{J K L} m_{K} m_{L}+\cdots
$$

The reference state is that of unit activity coefficient at infinite dilution of component $J$.
Considering a ternary mixture (components 1 , 2 , and 3 ) at constant temperature and substituting eq $\mathbf{B} \cdot 2$ into eq $\mathbf{B} \cdot 1$, we differentiate to obtain

$\frac{1}{R T}\left(\frac{\partial \mu_{3}}{\partial m_{2}}\right)_{P, m_{3}}=\beta_{23}+2 \gamma_{223} m_{2}+2 \gamma_{233} m_{3}+\cdots$

and

$\frac{1}{R T}\left(\frac{\partial \mu_{3}}{\partial m_{3}}\right)_{P, m_{2}}=\frac{1}{m_{3}}+\beta_{33}+2 \gamma_{233} m_{2}+2 \gamma_{333} m_{3}+\cdots$

Combining these results we find

$$
\frac{\left(\partial \mu_{3} / \partial m_{2}\right)_{P, m_{2}}}{\left(\partial \mu_{3} / \partial m_{3}\right)_{P, m_{3}}}=-\left(\frac{\partial m_{3}}{\partial m_{2}}\right)_{P, \mu_{3}}=\beta_{23} m_{3}+O\left(m_{3}{ }^{2}\right)
$$

so that

$$
\lim _{m_{3}=0}\left(\frac{\partial m_{3}}{\partial m_{2}}\right)_{P, \mu_{3}}=0
$$

and

$$
\lim _{m_{3} \rightarrow 0}\left[\frac{\partial}{\partial m_{3}}\left(\frac{\partial m_{3}}{\partial m_{2}}\right)_{P, \mu_{3}}\right]_{m_{2}}=-\beta_{23}
$$

This establishes the behavior of the interaction parameter $\lambda$ and its first derivative as $\phi_{3} \rightarrow 0$ inasmuch as $\partial \phi_{3} / \partial c_{2}$ is proportional to $\partial m_{3} / \partial m_{2}$ at this limit. Since

$$
\partial \phi_{3} / \partial c_{2}=-\partial \phi_{1} / \partial c_{2}
$$

the same argument, with interchange of subscripts 1 and 3 , shows that at the other limit, $\phi_{3}=1, \lambda$ is also zero and its derivative is finite.

\section{REFERENCES}

1. H. Shogenji, Busseiron Kenkyu, 62, 1 (1953); T. Ooi, J. Polym. Sci., 28, 459 (1958).

2. E. F. Casassa and H. Eisenberg, J. Phys. Chem., 64, 753 (1960); ibid., 65, 427 (1961); H. Eisenberg, J. Chem. Phys., 36, 1833 (1962).

3. A. Vrij, Dissertation, Utrecht (1959); A. Vrij and J. Th. G. Overbeek, J. Colloid Sci., 17, 570 (1962).

4. E. F. Casassa and H. Eisenberg, Advan. Protein Chem., 19, 287 (1964).

5. E. F. Casassa, Makromol. Chem., 150, 251 (1971).

6. P. Debye, J. Phys. Chem., 51, 18 (1947); B. H. Zimm, J. Chem. Phys., 16, 1093 (1948).

7. J. G. Kirkwood and R. J. Goldberg, ibid., 18, 54 (1950); W. H. Stockmayer, ibid., 18, 58 (1950). 
8. R. H. Ewart, C. P. Roe, P. Debye, and J.R. McCartney, ibid., 14, 687 (1946).

9. B. E. Read, Trans. Faraday Soc., 56, 382 (1960).

10. A. Dondos and H. Benoît, Makromol. Chem., 133, 119 (1970).

11. H. Margenau and G. M. Murphy, "The Mathematics of Physics and Chemistry,"' Second ed., D. Van Nostrand, 1965, Chapter 13.

12. A. Živný, J. Pouchlý, and K. Solč, Coll. Czech. Chem. Commun., 32, 2753 (1967).
13. J. M. G. Cowie, Pure Appl. Chem., 23, 355 (1970).

14. J. M. G. Cowie, R. Dey, and J. T. McCrindle, Polymer J., 2, 88 (1971).

15. C. Strazielle and H. Benoît, J. Chim. Phys., 58, 678 (1961).

16. G. V. Schulz, Z. Phys. Chem. B, 43, 25 (1939).

17. E. M. Barrall, M.J.R. Cantow, and J.F. Johnson, J. Appl. Polym. Sci., 12, 1373 (1968).

18. H. L. Wagner and C.A. J. Hoeve, J. Polym. Sci., Part A-2, 9, 1763 (1971). 